\title{
Case report; An adult presenting with bilateral hip joint dysplasia
}

Gunasekara $\mathrm{KS}^{1}$, Chinthaka $\mathrm{RG}^{1}$, Dissanayake $\mathrm{S}^{2}$

${ }^{I}$ Rheumatology and Rehabilitation unit, District General Hospital, Hambantota, ${ }^{2}$ Department of Anatomy, Faculty of Medical Sciences, University of Sri Jayewardenepura.

\section{Introduction}

Developmental dysplasia of the hip (DDH) describes a spectrum of conditions associated with the development of the hip in infants and young children. The spectrum includes abnormalities of stability (dislocation, subluxation) and abnormalities of shape of the femoral head and acetabulum (dysplasia). Dislocation and subluxation means complete loss of contact between the acetabulum and femoral head and partial loss while maintaining the contact between them respectively. Dysplasia encompasses various abnormalities of the shape of the hip joint usually shallow acetabulum. Normally DDH is diagnosed during infancy or early childhood following routine screening procedures or following consultations. Dysplasia without dislocation usually has no clinical findings and can be asymptomatic until adulthood. It is often found incidentally when radiographs are obtained for other concerns or when an adult presents with hip joint pain or abnormal gait. We are reporting a case of female patient diagnosed with dysplasia of the hip in her early thirties.

\section{Case report}

Mrs.B, 34 year old female presented to the Rheumatology unit at DGHHambantota with bilateral hip joint pain for one year duration. She was a housewife with a child of 3 year old. She described the pain as insidious in onset and progressive in nature mainly with weight bearing. Also pain was deep aching type in nature. Pain exacerbated with activities like walking and climbing a staircase and relived with rest. She did not give history of any traumatic event. She had memory of a similar episode of hip joint pain about 15 years ago which had lasted about 2 to 3 months but she could not recall any more details or did not have any documented evidence about investigations or treatment. During this presentation she did not have any other joint pain or swelling; no fever; no other specific complaints. She denied past medical or surgical problems and had normal uncomplicated vaginal delivery 3 years back. On examination she had normal gait with a mild limp. Left hip joint examination revealed restriction of all movements (flexion-40, abduction30 , adduction-20, internal rotation-20, external rotaion-30 degrees). Right side hip joint demonstrated reduced but to a lesser extent ompared to the left. (flexion-70, abduction-40, adduction30 , internal rotation-40, external rotation-40 degrees). There was no discrepancy in length of the leg. She did not have any other tender or swollen joints and all the other joint movements were in normal range. Subsequently she underwent few investigations which revealed no abnormality including 
normal inflammatory markers, negative rheumatoid factor, normal calcium level and thyroid functions. Then we arranged $\mathrm{x}$ rays of hip joints which are shown in picture 1,2 and 3. Finally it was diagnosed as bilateral acetabular dysplasia most probably developmental in origin with late presentation. Then we discussed with her and explain the nature of the condition and referred to the orthopedic unit for possible surgical correction.

\section{Discussion}

The hip joint is a ball and socket type synovial joint which facilitates wide range of movements without compromising stability. It is formed by the articulation of the femoral head with the acetabulum of the pelvis. All three constituents of the pelvic bone participate in forming the acetabulum; namely ileum forms the superior part, pubis forms the anterior and ischium forms the posteroinferior part. During foetal development by the $11^{\text {th }}$ week of gestation, the hip joint fully develops and the spherical femoral head deeply lie within the acetabulum. However, as the femoral head grows faster than the acetabulum, at the end of the gestation femoral head is incompletely covered by the acetabulum. Also during newborn period ligamentous laxity makes the developing hip susceptible to other external mechanical forces. These factors result in abnormal contact between the femoral head and the acetabulum. If this is not corrected abnormal hip joint contact can lead to structural anatomical changes like everted and flattened labrum, shallow acetabulum, abnormal ossification of the acetabulum [ 4 ] and inhibit the normal hip joint development. This can lead to various dysplastic changes of the hip joint with or without dislocation. This is known as Developmental Dysplasia of the Hip (DDH) which can range from symptomatic dislocation and instability to asymptomatic dysplsia. It is calculated that DDH has prevalence of 3 to 5 per 1000 children [ 1]. And it is more common among infants with certain risk factors (eg, female sex, breech position) [ 2].

The range of presentation of DDH can vary widely from instability during the newborn examination to subtle limited abduction in the infant, to asymmetric gait in the toddlers. When DDH is not detected and corrected early, patients may present with activity associated hip joint pain or hip joint osteoarthritis in the adulthood. Hip joint dysplasia without dislocation usually has no clinical findings and it could remain asymptomatic until early adulthood, which is the case with this patients. It is often found only through screening of risk factors or incidentally when radiographs are obtained for other concerns especially when investigating for activity related hip pain in an adolescent or young adult. Clinical course for patients with this presentation is variable but eventually it can lead to premature degenerative joint disease in hip joints. Pain may start soon after skeletal maturity or in females, during the pregnancy. Patients 40 to 60 years old who present with hip osteoarthritis are often found to have mild dysplasia as a contributing cause $[3,4]$. 
So it is important to identify every form of $\mathrm{DDH}$ as soon as possible to initiate early corrective measures in order to prevent permanent abnormalities and deformities. Every infant soon after the birth should undergo thorough clinical examinations of the hip joints to identify any dislocatability. Any infant with suspicion should be directed to further investigations. Ultrasonography is the primary imaging technique for assessing the morphology and stability of the infant hip. Place of $X$ rays of the hip joints in evaluating DDH depends upon the age of the patient and the degree of ossification. Plain radiographs have limited value in the detection of DDH during the first six months of life when the femoral head and acetabulum are unossified. Children after the age of six months and in adults plain radiographs are useful in the evaluation for DDH [ 5 ].Lateral and superior positioning of the ossified portion of the femoral head and neck is an important finding consistent with DDH.

Once diagnosed treatment of DDH is initiated with referral to a orthopedic surgeon. The goals of treatment are to obtain and maintain concentric reduction of the hip to provide an optimal environment for the development of the femoral head and acetabulum. Treatment option depends on the age at which DDH is diagnosed. Infants younger than six months who have dislocated or persistently have the potential to dislocate hips are usually treated with abduction splinting. Children older than this require closed or open reduction under anesthesia. Undiagnosed dysplasia may result in progression to premature degenerative joint disease ultimately requiring surgical interventions including hip joint replacement during adulthood.

This case emphasizes the importance of neonatal clinical assessment of the hip joints especially those with risk factors for DDH.

Dr Kusala S. Gunasekara, Consultant Rheumatologists, District General Hospital, Hambanthota.

kusalagunasekara@yahoo.com

Submitted by - June 2019

Accepted by - July 2019

\section{References}

1. Bialik V, Bialik GM, Blazer S, et al. Developmental dysplasia of the hip: a new approach to incidence. Pediatrics 1999; 103:93.

2. Shipman SA, Helfand M, Moyer VA, Yawn BP. Screening for developmental dysplasia of the hip: a systematic literature review for the US Preventive Services Task Force. Pediatrics 2006; 117:e557.

3. Wedge JH, Wasylenko MJ. The natural history of congenital dislocation of the hip: a critical review. Clin Orthop Relat Res 1978; $: 154$.

4. Wedge JH, Wasylenko MJ. The natural history of congenital disease of the hip. J Bone Joint Surg Br 1979; 61-B:334. 
5. American College of Radiology. ACR Appropriateness Criteria. Developmental dylsplasia of the hip-child.

\section{Figures}

Figure 1. X ray Pelvis including bilateral hip joints

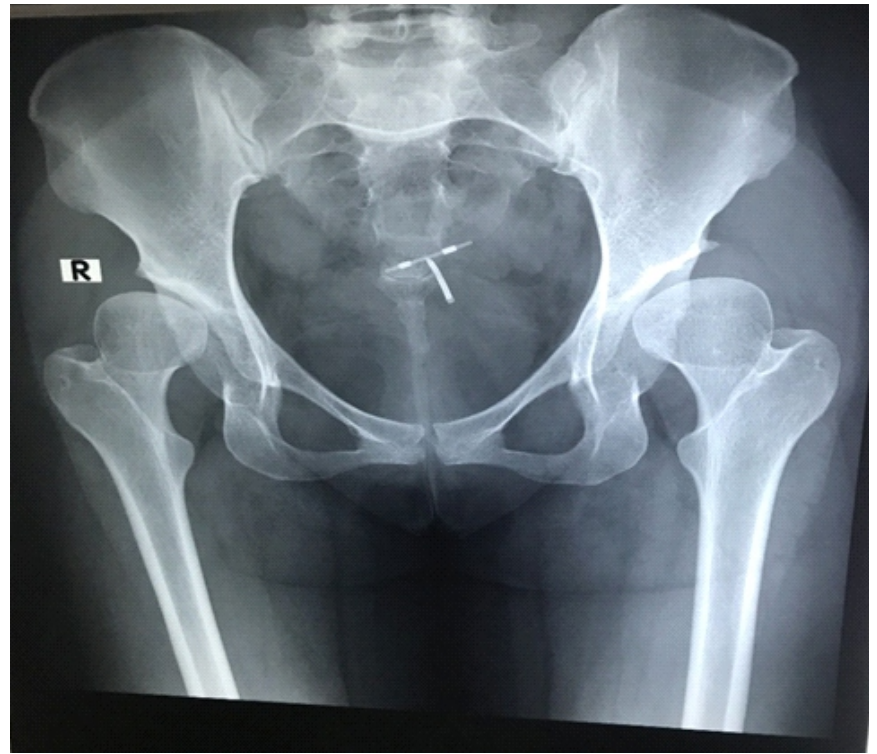

Picture $-2-X$ ray view of left hip joint

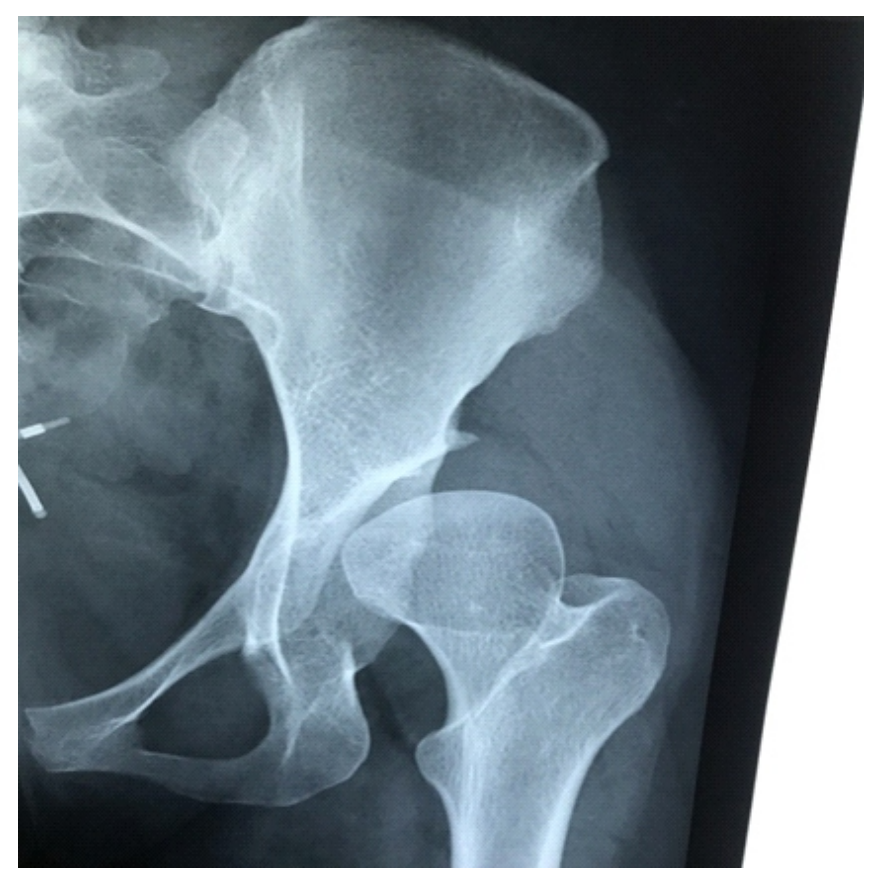


Gunasekara KS, Chinthaka RG, Dissanayake S - An adult presenting with bilateral hip joint dysplasia

Picture $3-X$ ray view of right hip joint

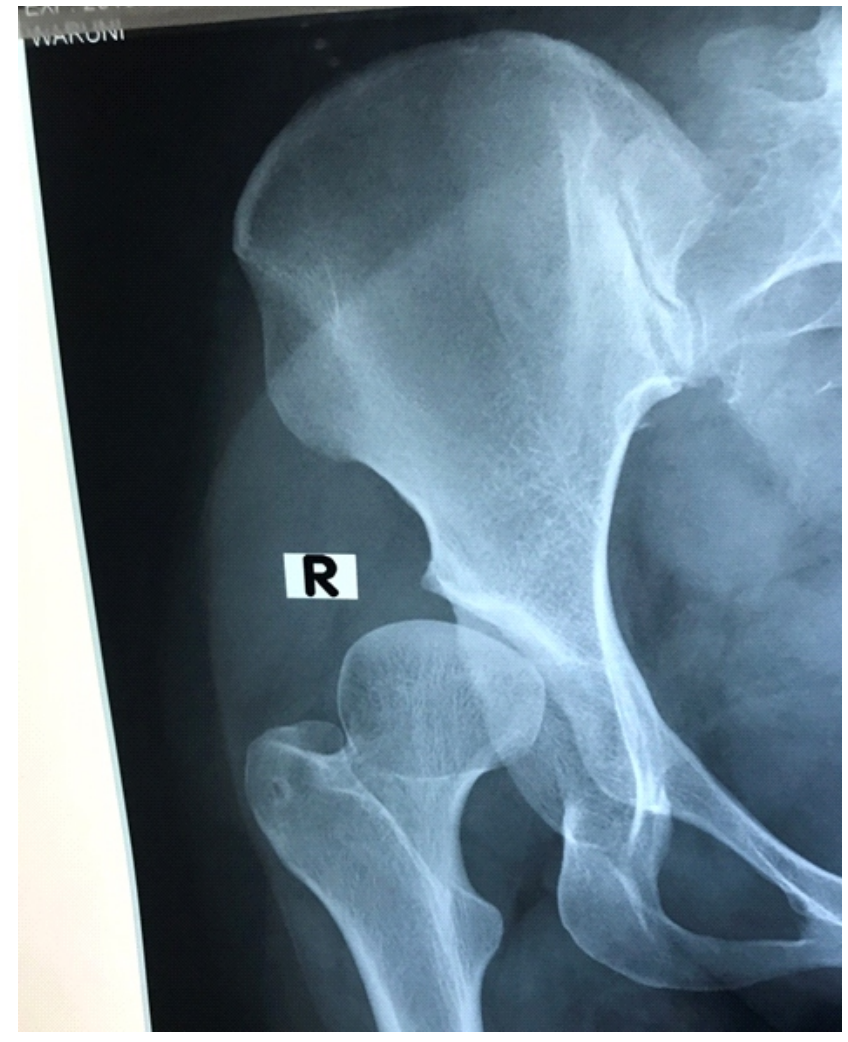

